\title{
Differentiation of Neisseria gonorrhoeae IB-3 and IB-7 serovars by direct sequencing of protein IB gene and pulsed-field gel electrophoresis
}

\author{
C. L. POH, O. C. LAU AND V. T. K. CHOW \\ Department of Microbiology, Faculty of Medicine, National University of Singapore, Kent Ridge, \\ Singapore 0511, Republic of Singapore
}

\begin{abstract}
Summary. Serotyping of Neisseria gonorrhoeae based on the differential recognition by a panel of six monoclonal antibodies (MAbs) against Protein IB (PIB) is a valuable tool for studying the epidemiology of gonorrhoea. However, the predominance of certain serovars in specific geographic regions necessitates the development of new MAbs or new genotyping approaches. Nucleotide and amino-acid sequence analysis of PIB from strains within the IB-3 and IB-7 serovars revealed strain differences within the same serovar. Based on the generation of PIB nucleotide and deduced amino-acid sequences that centred on amino-acid residues 196 and 237, eight serovar IB-3 strains and nine serovar IB-7 strains were separately subdivided into five groups. Intra-serovar differences were also established by pulsed-field gel electrophoresis (PFGE) of macro-restriction fragments generated by SpeI- and NheI-cleavage of genomic DNA. There was good correlation between the results based on PIB gene PCRsequencing and those based on PFGE analysis of macro-restriction fragment patterns. These data demonstrate the high precision of PFGE analysis and indicate that this approach can be used as a rapid epidemiological subtyping system for major serovars of $N$. gonorrhoeae.
\end{abstract}

\section{Introduction}

Neisseria gonorrhoeae infection is one of the most common sexually transmitted diseases worldwide. Progress in vaccine development has been slow and the mainstay in the prevention of $N$. gonorrhoeae infection has been through epidemiological surveillance and antimicrobial treatment of infected individuals. ${ }^{1}$ Epidemiological surveillance involves contact tracing, screening of high-risk, asymptomatic individuals in the community and the development of accurate strain characterisation markers to determine the spread of specific strains. The auxotype-serovar (A/S) classification is currently the most widely accepted method for the discrimination of $N$. gonorrhoeae isolates. ${ }^{2}$ However, the A/S classification system has some major limitations because different strains often express the same auxotype. ${ }^{3}$ Serotyping provides a far more sensitive method for strain discrimination than auxotyping but there is scepticism that it may over-discriminate some serovars, while not providing sufficient discrimination of other strains within major serovars such as IB-1 and IB-3. ${ }^{4}$

Comparison of the entire nucleotide sequence of gonococcal genomes may lead to a definitive typing method although this approach is relatively complex, expensive and impractical for large numbers of isolates. A simpler approach involves amplification, by the polymerase chain reaction (PCR), and direct DNA sequencing of a single gene, e.g., the $N$. gonorrhoeae protein IB (PIB) gene, which encodes serovar specificity. ${ }^{5}$ Alternatively, pulsed-field gel electrophoresis (PFGE) of gonococcal genomic DNA digested by low-frequency cleavage restriction endonucleases has been used to characterise $N$. gonorrhoeae. ${ }^{6}$ This approach examines the entire genome of isolates and differences in nucleotide sequences are reflected in the resulting DNA fingerprints. Since PFGE provided useful differentiation of common serovars in a previous study, ${ }^{6}$ the present study compared this method with PCR sequencing of the hypervariable region of the PIB gene for characterising $N$. gonorrhoeae strains.

\section{Materials and methods}

\section{Bacterial isolates}

Seventeen epidemiologically unrelated $N$. gonor- 
a

IB3. 58

IB3. 533

IB3.S12

IB3.S25

IB3.S31

IB3. 540

IB3. 552

IB3.S21

IB3. 58

IB3.S33

IB3.S12

IB3. 525

IB3.S31

IB3. 540

IB3.S52

IB3. 521

IB3. 58

IB3.S33

IB3. 512

IB3. 525

IB3.S31

IB3. 540

IB3. 552

IB3.S21

IB3. 58

IB3. 533

IB3. 512

IB3.S25

IB3.S31

IB3. 540

IB3 . 552

IB3. $\mathbf{S 2 1}$

IB3. 58

IB 3.533

IB3.S12

IB3. 525

IB3. $\$ 31$

IB3. 540

IB3. 552

IB3.S21

IB 3.58

IB3.S33

IB3. $\mathrm{S} 12$

IB3. 525

IB3.S31

IB3. 540

IB3. 552

IB3. S21

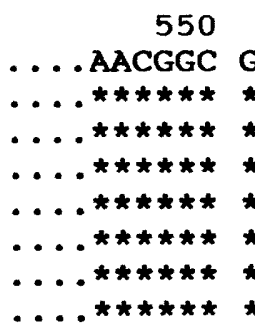

600

TCTTCGCACA

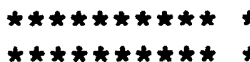

$\star \star \star \star * * * * * * \star$

$\star \star \star \star \star \star \star \star \star * \star * *$

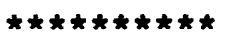

$\star * \star * * * * * * *$

$\star \star * * * * * * * * *$

650

AAAATCGAAT

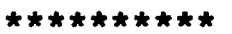

$* * * * * * * * * *$

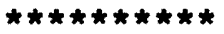

$\star \star \star \star * \star * \star * * * * *$

$\star * * * * * * * * *$

$\star \star \star \star \star \star * \star * * * *$

$\star \star \star \star * \star G * * *-$

700

AAAACTGCAA GT

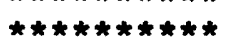

$\star \star \star \star \star \star \star \star * \star * \star *$

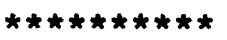

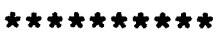

$* \star * * \star * \star * * * *$

$\star \star \star * * * * * * *$

750

ACGTTTCCGT

$\star \star \star \star \star \star \star \star \star \star \star \star *$

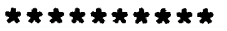

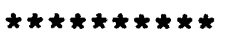

$\star \star \star * * * * * * * *$

$\star \star * * * * * * * * *$

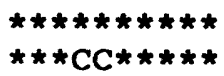

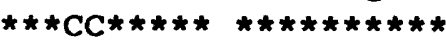

804

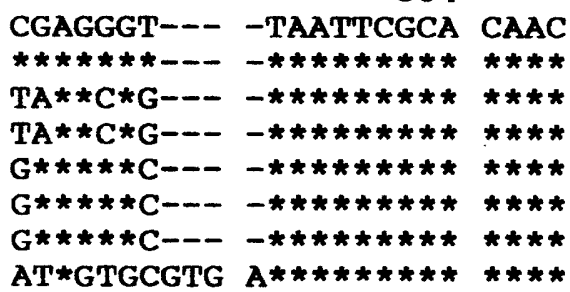

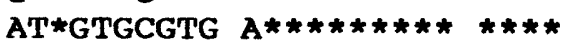

$\star \star \star \star \star \star \star \star \star \star \star \star \star \star \star * t * t$

660

$\star * \star * \mathrm{ACA} * * *$

$-* \star A T *$
$560 \quad 570$

580

590

GAATCTTACC ACGTTGGCTT GAACTACCAA AACAGCGGCT

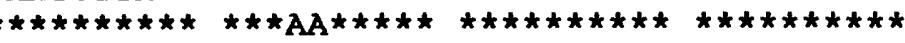

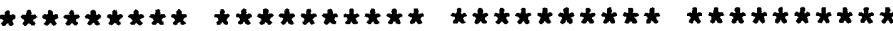

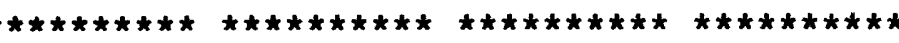

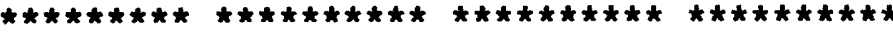

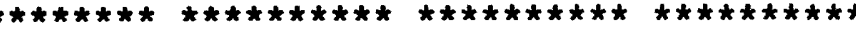

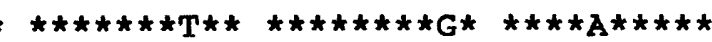

620
TTGTTCCAAA

630

640

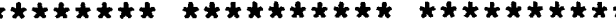

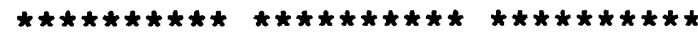

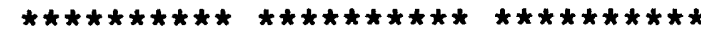

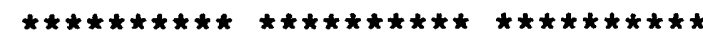

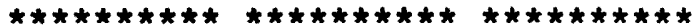

670

680

690

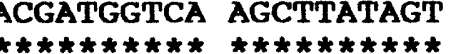

ATGCCCAGTC TGTTTGTTGA

$\star \star C * * * * * C * * * * * * * * * * *$

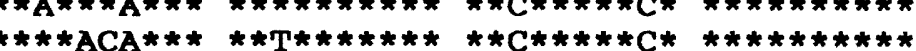

$\star * \star * A C A * * * * * \mathrm{~T} * * * * * * * * * \mathrm{C} * * * * * \mathrm{C} * \star * * * * * * * * * *$

$* T_{*} * * * * * *$

$T * * A * * * * A *$

$\star * C * * * * * C * * * * * * * * * * *$

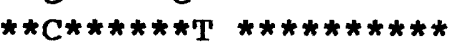

720

730

740

GTTCACCGTT TGGTAGGCGG TTACGACAAT AATGCCCTGT

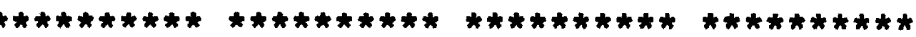

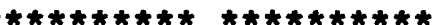

$\star \star \star \star \star \star \star * \star * \star * * *$

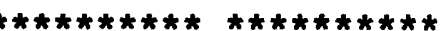

$* * * * * * * * * * * * * * * * * * * *$

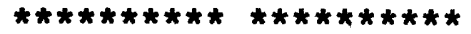

$\star * * * * * * * * * * * * * * * * * * *$

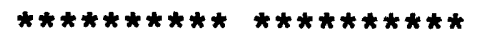

$\star * * * C * * * * *$

\section{0}

780

788

CAACAAGATG CCAAATTGTA T--ggagcaA

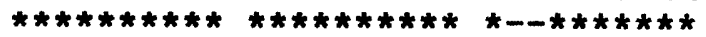

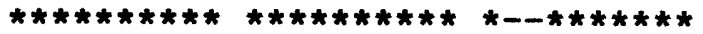

$\star * * * * * * * * * * * * * * * * * * * *--* * * * * * *$

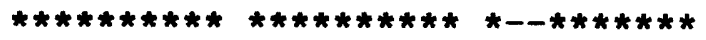

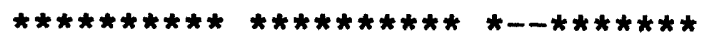

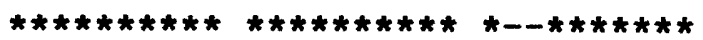

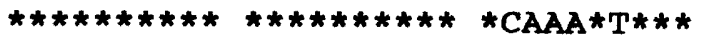

Fig. 1. For legend see facing page

rhoeae strains comprising eight IB-3 and nine IB-7 serovars were studied. These strains were part of a collection of 48 clinical strains previously isolated from patients attending a local hospital from June to August $1986 .{ }^{6}$ The strains were auxotyped and serotyped by Drs S. Sarafian and J. S. Knapp, Centers for Disease Control, Atlanta, GA, USA. ${ }^{4}$

\section{PFGE}

PFGE was performed with the contour-clamped homogeneous electric field apparatus (CHEF-DRII; BioRad, Richmond, CA, USA) as described previously. ${ }^{6}$ Briefly, genomic DNA samples in agarose plugs (In Cert Agarose; FMC, Rockland, ME, USA) 
b

IB3. 58

IB3. 533

IB3.S12

IB3.S25

IB3.S31

IB3. 540

IB3. 552

IB3.S21

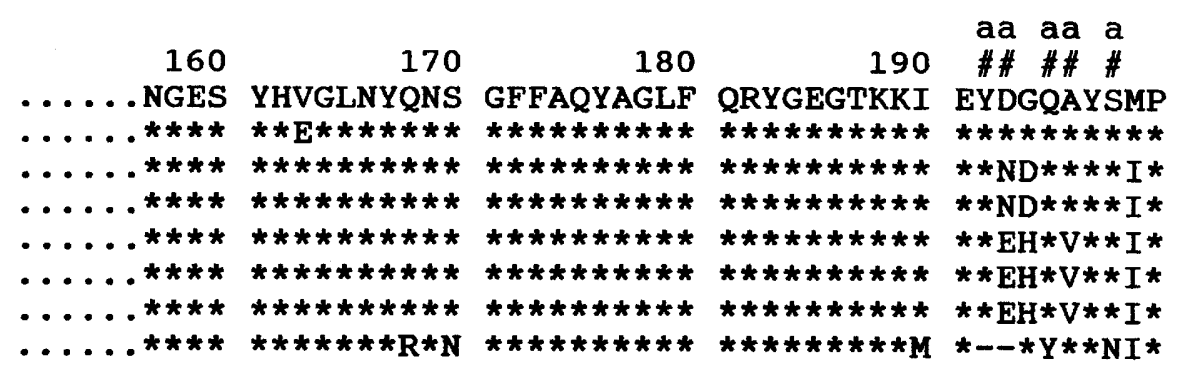

210

220

230

hhhhh $\mathrm{h}$

\#\#\#\# \#

SLFVEKLQVH RLVGGYDNNA LYVSVAAQQQ DAKLYGAT-- RVNSHN

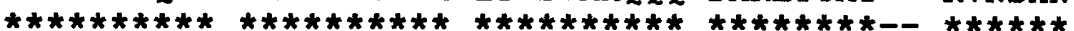

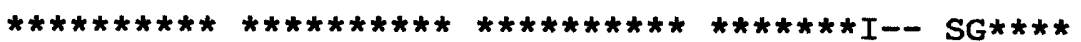

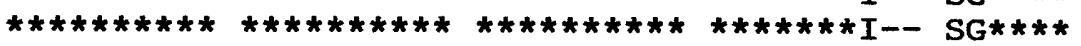

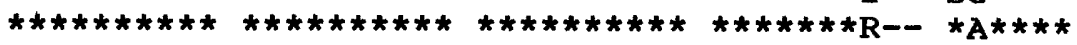

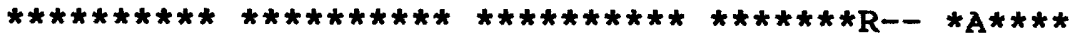

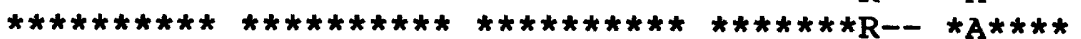

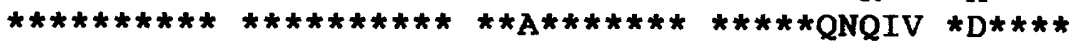

IB3.S52

Fig. 1. a, Comparative nucleotide sequence analysis of PIB gene fragments of serovar IB-3 strains. The numbering is according to Butt $e t$ al ${ }^{\text {? }}$ *: identity; - : deletion. b, Comparison between deduced amino-acid sequences of the protein IB $^{8}$ of serovar IB-3 strains. ${ }^{*}$ : identity; - : deletion; $\mathrm{a}$ : recognised by MAb 3C8; h: recognised by MAb 2D4.

were digested with SpeI or NheI (New England Biolabs, Beverly, MA, USA) overnight at $37^{\circ} \mathrm{C}$ in $150 \mu \mathrm{l}$ of restriction endonuclease buffer containing $10 \mathrm{U}$ of endonuclease. Digested plugs were electrophoresed in agarose $1 \%$ gels (SeaKem GTG agarose, FMC) for $22 \mathrm{~h}$ with pulse times of 1-15 s. Gels were stained with ethidium bromide, destained in water, and photographed under UV illumination.

\section{$P C R$ and direct cycle sequencing}

Extraction of bacterial DNA and PCR amplification of a 341-bp fragment (nucleotides 519-859) of the variable region of the $N$. gonorrhoeae PIB gene with OMG1 and OMG2 primers were as described previously. ${ }^{5}$ The PCR products were purified and their DNA sequences were determined in both directions by direct cycle sequencing with ${ }^{32} \mathrm{P}$-labelled OMG1 and OMG2 primers as sequencing primers, as described previously. ${ }^{5}$ The resultant nucleotide sequences were converted to their respective amino-acid sequences by the DNASIS program, and then aligned for maximum homology with the PROSIS program (Hitachi, Brisbane, CA, USA).

\section{Results}

Nucleotide and deduced amino-acid sequences of $P I B-3$ and PIB-7 serovar strains

Comparison of the nucleotide sequences of the eight IB-3 strains revealed diversity centred around two discrete regions corresponding to nucleotides $646-680$ and 782-795 (fig. 1a). ${ }^{7}$ Analysis of the deduced aminoacid sequences of the eight strains indicated changes within codons 190-199 and 236-240 (fig. 1b) ${ }^{8}$ Comparison of nucleotide sequences of the nine IB-7 strains also indicated differences mainly at positions $646-680$ (fig. 2a) corresponding to amino-acid changes at codons 190-199 (fig. 2b).

Comparing the DNA sequences of the eight IB-3 strains showed that there were 2-36 nucleotide differences. Of the maximum number of 36 nucleotide changes, six were silent. Strains S8 and S33 differed in only two nucleotides whereas strain $\mathbf{S} 21$ differed from strains S31, S40 and S52 at 36 nucleotide positions. The deduced changes in amino-acid residues varied from a single difference (between strains S8 and S33) up to 16 differences (between strains S21 and S33). The eight IB-3 strains were subdivided into five groups based on nucleotide and amino-acid sequences. The distribution of strains into their respective groups based on nucleotide sequences was found to concur with that based on amino-acid sequences (table I).

Amongst the nine IB-7 strains, only two nucleotide differences were observed between strains S22 and S43; the highest number of 27 nucleotide differences was found between strains S1 and S43, five of these being silent changes. Comparison of deduced aminoacid residues of the nine IB-7 strains revealed from two amino-acid changes (between strains S22 and S43) up to 13 changes (between strains S1 and S19). Based on nucleotide and deduced amino-acid sequences, the nine IB-7 strains were subdivided into five groups. With the exception of a single group comprising five strains, the remaining four groups each consisted of a single isolate. There was perfect correlation between the grouping of IB-7 strains based on nucleotide and amino acid sequences (table II).

\section{PFGE}

Restriction endonucleases SpeI and NheI were found to generate PFGE profiles that were easily read. 
a

IB7. 543

IB7. 522

IB7. 526

IB7. 528

IB7. 530

IB7. 541

IB7. 550

IB7. 519

IB7. S1

IB7 . S4 3

IB7. 522

IB7. 526

IB7. $\mathbf{5 2 8}$

IB7. 530

IB7. 541

IB7. 550

IB7. 519

IB7. S1

IB7. 543

IB7. 522

IB7. 526

IB7. 528

IB7. 530

IB7. 541

IB7. 550

IB7. S19

IB7.S1

IB7. S4 3

IB7. 522

IB7. 526

IB7. $S 28$

IB7. S30

IB7.S41

IB7. 550

IB7. $\$ 19$

IB7. S1

IB7. 543

IB7. 522

IB7. S26

IB7. 528

IB7. 530

IB7. 541

IB7. 550

IB7 . 519

IB7.S1

IB7. S4 3

IB7.S22

IB7. 526

IB7. 528

IB7. 530

IB7. 541

IB7. 550

IB7. S19

IB7. $\mathrm{S1}$
550

560

570

580

590

....AACGGC GAATCTTACC ACGTTGGTTT GAACTACCGA AACAACGGCT

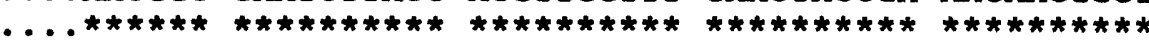

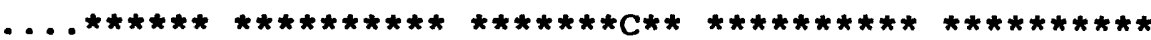

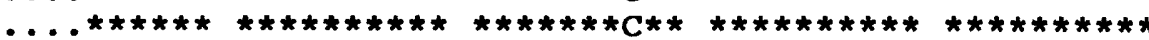

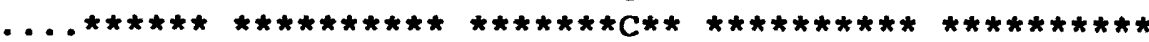

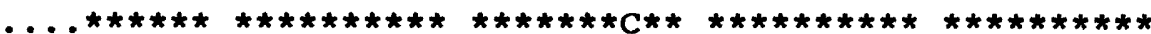

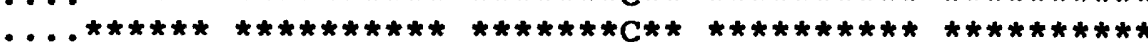

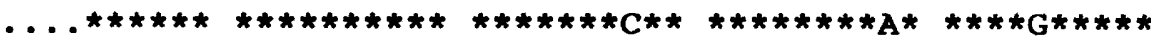

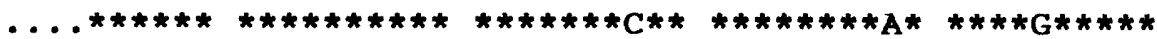

600 TCTTCGCACA $\star \star \star * * * * * * * *$ $\star * * * * * * * * *$ $\star * * * * * * * * *$ $* * * * * * * * * *$ $\star \star * * * * * * * *$ $\star \star \star * * * * * * * *$ $\star \star \star \star * * \star * * * \star *$

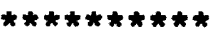

610 ATACGCCGGC TTGTTCCAAA

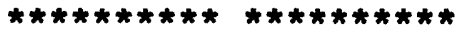

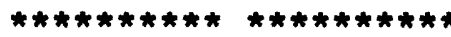

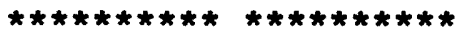
$* \star * * * * * * * * * * * * * * * * * *$ $\star * * * * * * * * * * * * * * * * * * * * *$

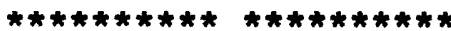

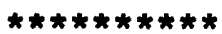

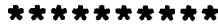

$\star * * * * * * * * *$

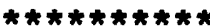

AAAATCGAA $\star \star \star \star \star * \star * \star * \star-$ $\star \star * * * \mathrm{G} * * *-$ $\star * \star * * \mathrm{G} * * *-$ $\star \star \star \star \star \star G \star * \star \star-$ $\star \star \star \star \star \star G * \star * *-$ $\star \star \star * \star * G * * *-$ $* * * * * \mathrm{G} * * *-$ $\star \star \star \star \star \star * \star * * T$

\section{0}

AAAACTGCAA $\star \star \star \star \star \star * * * * *$ $\star \star \star * \star * \star * \star * * \star$ $\star * * * * * * * * *$ $\star \star \star * \star * \star * * * * *$ $\star \star * * * * * * * *$ $\star \star \star * * * * * * * \star$ $\star \star \star * \star * * * * * *$

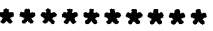

750 ACGCCTCCG' $\star \star \star \star \star * \star * \star * \star * *$ $\star \star \star * * * * * * * *$ $\star * \star * \star \star * * * \star * *$ $* * * * * * * * *$ $\star \star * * * * * * * *$ $\star * * * * * * * * *$ $\star \star \star \star \star \star \star \star \star * \star * *$

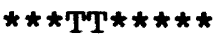

800 CGTGCTAATT CGCACAACTC $\star \star \star * \star * \star \star * * *$ $\star * \star * * * * * * *$ $\star * * * * * * * * *$ $\star \star \star * * * * * * * *$ $\star \star * * * * \star * \star *$

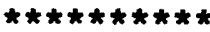

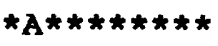
$\mathrm{A} * \mathrm{G} * \mathrm{~T} * \star \star \star \star \star$ $----* * *{ }^{2} \mathrm{C} *$ $\star * * * * * * * * * * * * * * * * * * * t$

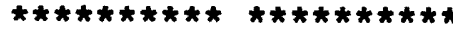

670 GGCTA TCAGTATAAT $* * * C * * * * * * * * * *$ $----* * A * *$ $----\cdots * A * *$ $----* * A * *$ $-----* * A * *$ -GGAT-AT** $\star A C A * * * * \star *$ $\star \mathrm{ACA} * * * * * *$

$\star \mathrm{ACA} * * * * * *$ $* \mathrm{ACA} * * * * * *$

$* \mathrm{ACA} * * * * * *$ *TPA****** ACGAT**TC*
AGCT****G*
630

640
CGGCGA AGGCACTAAA

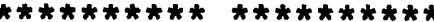
$\star * * * * * * * * * * * * * * * * * * *$

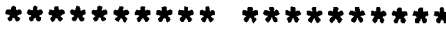
$\star * * * * * * * * * * * * * * * * * * *$ $\star \star * * * * * * * * * * * * * * * * * * *$

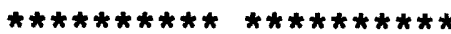
$\star \star \star \star \star \star \star \star * \star * \star * * * * * * * * * * * *$

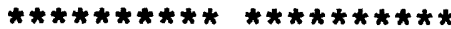

$680 \quad 690$
AGCCCCAGTC TGTTTGTTGA $\star \mathrm{T} * \star * \star * \star * * * * * * * \star * * * * *$

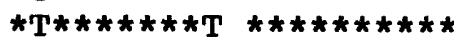

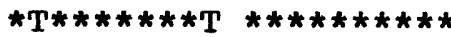

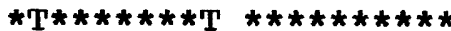

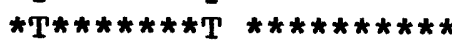

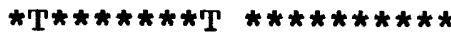

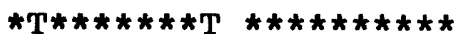

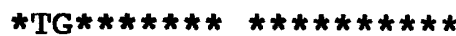

$\begin{array}{llll}710 & 720 & 730 & 740\end{array}$

GTTCACCGTT TGGTCGGCGG TTACGACAAT AATGCCCTGT

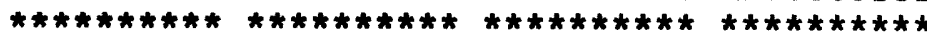

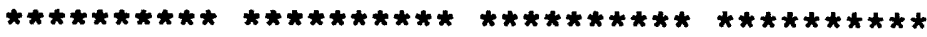

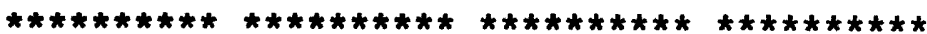

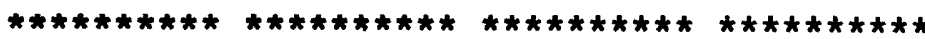

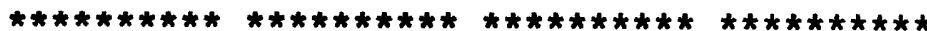

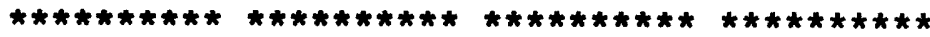

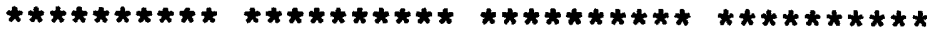

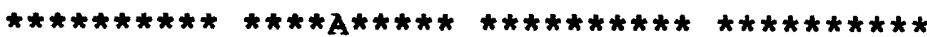

$760 \quad 770 \quad 780 \quad 790$
agcCgcacaA caAcaAgatg CCAAATTGTA TGgaACATGg

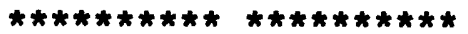

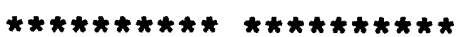

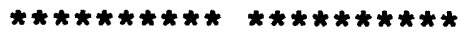

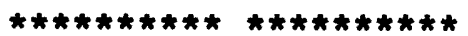

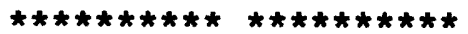

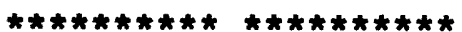

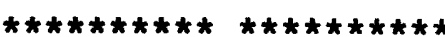

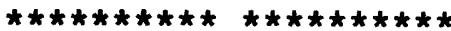

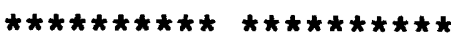

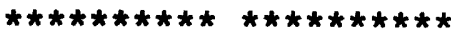

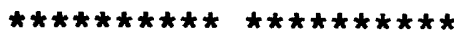

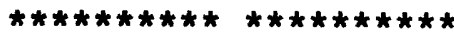
$\star * * * * * * * * * \star * * * * \mathrm{G} * * \mathrm{AC} *$

CGCACAACTC TCAAACCGAA GTTGCCGCTA CC

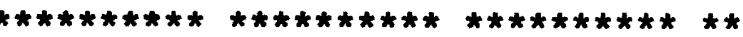

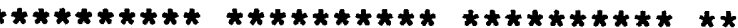

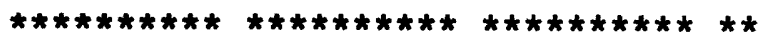

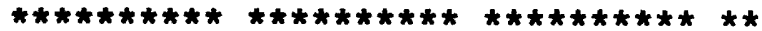

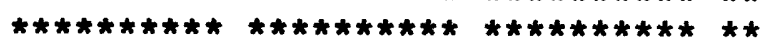

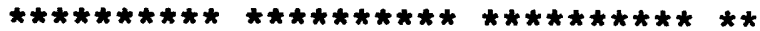

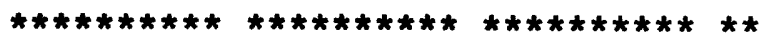

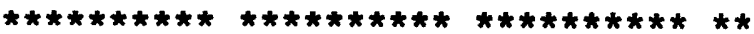

Fig. 2. For legend see facing page. 
b

IB7. 543

IB7. 522

IB7. 526

IB7 . 528

IB7. 530

IB7. 541

IB7. $\$ 50$

IB7.S19

IB7 : SI

IB7. 543

IB7.S22

IB7. 526

IB7. 528

IB7. 530

IB7. 541

IB7. $S 50$

IB7. $S 19$

IB7. $\mathrm{S} 1$

\begin{tabular}{|c|c|c|c|c|}
\hline & 170 & 0 & 180 & $\begin{array}{l}\text { aa } \\
\text { aa } \\
\# \text { \# }\end{array}$ \\
\hline $\begin{array}{l}\text { NG } \\
* *\end{array}$ & $\begin{array}{l}\text { YHVGLNYRNN } \\
\star \star \star \star * * * * * *\end{array}$ & $\begin{array}{l}\text { GFFAQYAGLF } \\
\star * \star * \star * \star * * *\end{array}$ & $\begin{array}{l}\text { QRYGEGTKKI } \\
\star * \star * * * * * \star *\end{array}$ & $\begin{array}{l}\text { E--GYQYNSP } \\
\star--\star H^{*} \star * I *\end{array}$ \\
\hline .** & $\star \star \star \star \star \star \star \star \star \star * \star * \star$ & $\star \star \star \star \star \star * * * \star * \star * \star$ & $\star \star \star \star \star \star \star \star \star \star * \star * M$ & $\star--\star * t * * I *$ \\
\hline & $\star * * * * * * * * *$ & $\star * * * * * * * * *$ & $\star \star \star \star \star * \star * \star * \star M$ & $T * \star I$ \\
\hline $\begin{array}{l}* \star \star \star \star \\
. * \star * \star\end{array}$ & 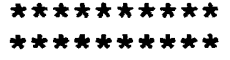 & 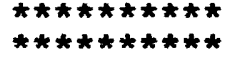 & 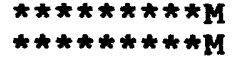 & $\begin{array}{l}\star--* * T * * I * \\
\star--* * T * I *\end{array}$ \\
\hline $\begin{array}{l}* \star * \star * \\
* \star \star * \\
* \star * \star\end{array}$ & 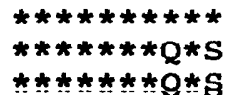 & 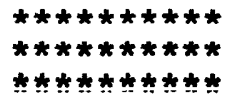 & $\begin{array}{l}\star * * * * * * * * \mathbf{M} \\
\star * * * * * * * * \mathbf{M} \\
* * * * * * * * * t\end{array}$ & $\begin{array}{l}\star--\star * T * * I * \\
*-G Y * L * I * \\
\star Y B \neq \Omega A \star S M\end{array}$ \\
\hline
\end{tabular}

$\begin{array}{llllll} & & \text { hhhhh } & h & \\ & 220 & 230 & \# \# \# \# & \# & 248\end{array}$ SLFVEKLQVH RLVGGYDNNA LYASVAAQQQ DAKLYGTW-- RANSHNSQTE VAAT

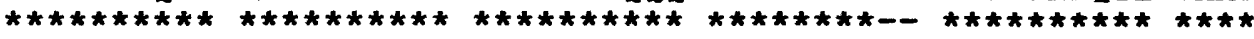

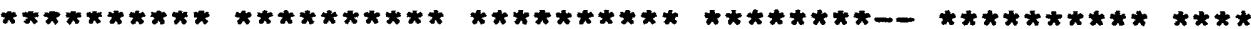

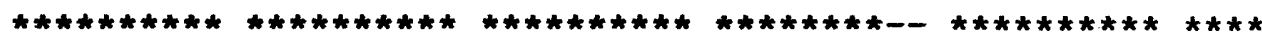

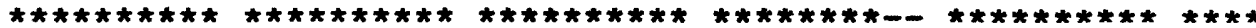

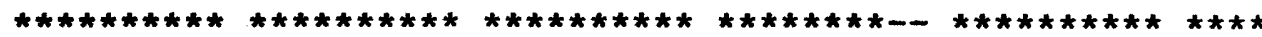

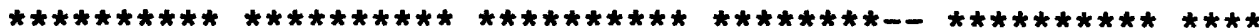

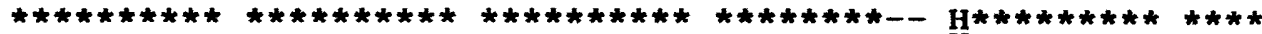

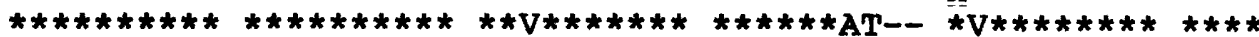

Fig. 2. a, Comparative nucleotide sequence analysis of PIB gene fragments of serovar IB-7 strains. *: identity; - : deletion. b, Comparison between deduced amino-acid sequences of the protein IB of serovar IB-7 strains. *: identity; - : deletion; a: recognised by MAb 3C8; h: recognised by MAb 2D4.

Table I. $N$. gonorrhoeae IB-3 serovar strains analysed by auxotyping, PFGE of SpeI and NheI macro-restriction fragments and PIB nucleotide and amino-acid sequences

\begin{tabular}{llccccc}
\hline $\begin{array}{c}\text { Strain } \\
\text { no. }\end{array}$ & Auxotype & $\begin{array}{c}\text { SpeI } \\
\text { pattern }\end{array}$ & $\begin{array}{c}\text { NheI } \\
\text { pattern }\end{array}$ & $\begin{array}{c}\text { Nucleotide } \\
\text { sequence } \\
\text { pattern }\end{array}$ & $\begin{array}{c}\text { Amino-acid } \\
\text { sequence } \\
\text { pattern }\end{array}$ & $\begin{array}{c}\text { GenBank } \\
\text { no. }\end{array}$ \\
\hline S8 & Proline & 1 & 1 & 1 & 1 & U17238 \\
S40 & Proline & 2 & 2 & 2 & 2 & U17029 \\
S12 & Prototrophic & 3 & 3 & 3 & 3 & U17020 \\
S21 & Prototrophic & 4 & 4 & 4 & 4 & U17022 \\
S25 & Prototrophic & 3 & 3 & 3 & 3 & U17023 \\
S31 & Prototrophic & 2 & 5 & 2 & 2 & U17027 \\
S33 & Prototrophic & 5 & 6 & 5 & 5 & U17028 \\
S52 & Prototrophic & 6 & 7 & 2 & 2 & U17237 \\
\hline
\end{tabular}

Table II. $N$. gonorrhoeae IB-7 serovar strains analysed by auxotyping, PFGE of SpeI and NheI macro-restriction fragments and PIB nucleotide and amino-acid sequences

\begin{tabular}{clccccc}
\hline $\begin{array}{c}\text { Strain } \\
\text { no. }\end{array}$ & Auxotype & $\begin{array}{c}\text { SpeI } \\
\text { pattern }\end{array}$ & $\begin{array}{c}\text { NheI } \\
\text { pattern }\end{array}$ & $\begin{array}{c}\text { Nucleotide } \\
\text { sequence } \\
\text { pattern }\end{array}$ & $\begin{array}{c}\text { Amino-acid } \\
\text { sequence } \\
\text { pattern }\end{array}$ & $\begin{array}{c}\text { GenBank } \\
\text { no. }\end{array}$ \\
\hline S1 & Proline & 1 & 1 & 1 & 1 & U17019 \\
S19 & Proline & 2 & 2 & 2 & 2 & U17021 \\
S26 & Proline & 3 & 3 & 3 & 3 & U17024 \\
S28 & Proline & 3 & 3 & 3 & 3 & U17025 \\
S30 & Proline & 3 & 3 & 3 & 3 & U17026 \\
S41 & Proline & 3 & 3 & 3 & 3 & U17234 \\
S43 & Proline & 4 & 4 & 4 & 4 & U17235 \\
S22 & Prototrophic & 5 & 5 & 5 & 5 & U09044 \\
S50 & Prototrophic & 6 & 6 & 3 & 3 & U17236 \\
\hline
\end{tabular}

As shown in fig. 3a, each SpeI fingerprint of IB-3 strains contained 13-17 fragments, ranging from 2 to $350 \mathrm{~kb}$. Digestion with NheI produced 14-16 fragments, ranging from 20 to $410 \mathrm{~kb} .{ }^{6}$ SpeI and NheI digestion of genomic DNA samples from the eight
IB-3 strains revealed six and seven DNA patterns, respectively (table I). Amongst the IB-7 strains, SpeI and NheI analysis each generated six patterns (fig. $3 b$ ), ${ }^{6}$ and the distribution of strains into their respective patterns correlated highly (table II). 
a

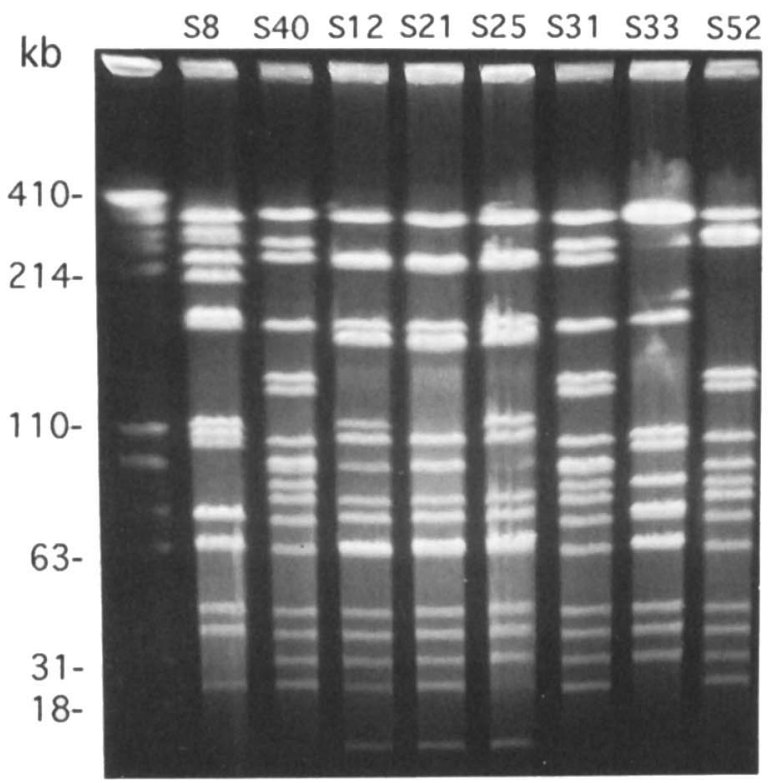

b

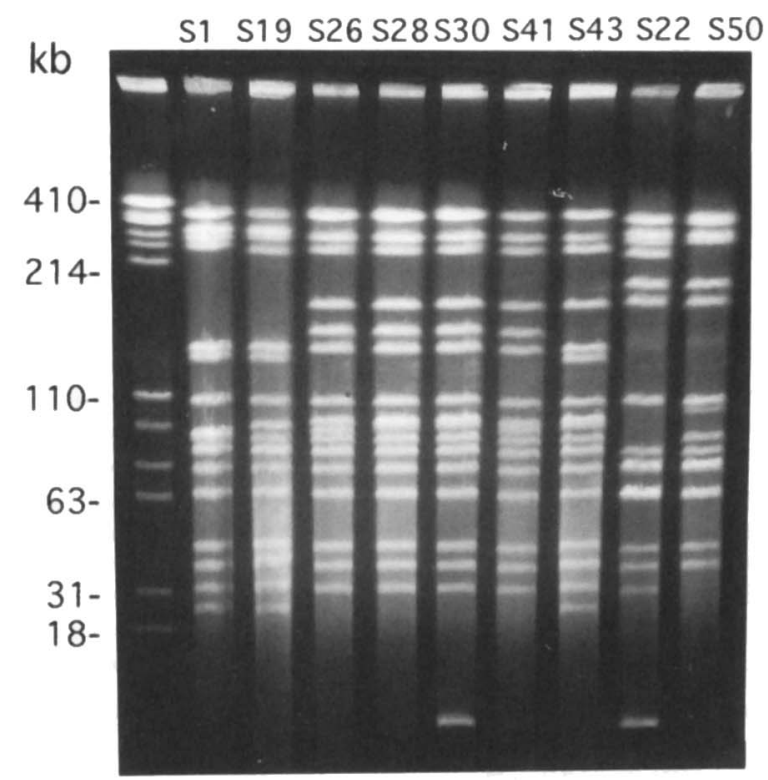

Fig. 3. PFGE analysis of SpeI-cleaved genomic DNA samples of $N$. gonorrhoeae: $\mathbf{a}$, IB-3 strains; $\mathbf{b}$, IB-7 strains. The strain numbers are given above each track. Rhodobacter sphaeroides 2.4.1 digested with AseI served as the size marker (track 1$)$.

\section{Discussion}

PFGE resolution of chromosomal macro-restriction fragments has been shown to be a highly discriminatory tool for the delineation of genetic relatedness of bacterial pathogens. ${ }^{9-11}$ In the present study, PFGE analysis of SpeI and NheI macro-restriction fragments generally correlated well with direct PCR sequencing of PIB gene, albeit with some discrepancies. For example, the IB-3 serovar strains S40, S31 and S52 showed identical nucleotide and amino-acid sequences but the three strains were subtyped into two SpeI and three NheI patterns. Of the nine strains belonging to serovar IB-7, four strains (S26, S28, S30 and S41) were found to have identical PFGE profiles, as well as nucleotide and deduced amino-acid sequences. The only discordance was found with strain S50 which was in the same nucleotide and amino-acid group as strains S26, S28, S30 and S41 but exhibited both SpeI and NheI PFGE patterns that differed significantly from these strains. The occurrence of different PFGE profiles in strains having identical PIB nucleotide and amino-acid sequences could be explained by the divergence in nucleotide sequences at other sites of the genome through either point mutations or genome rearrangements. If such genetic events were to occur in the PIB gene itself, and if the mutations were to affect the overall epitope conformation, serovar changes could be expected. An alternative possibility, especially in a naturally transformable organism like $N$. gonorrhoeae, could be that this PIB gene may have been acquired by strain $\mathrm{S} 50$ by genetic recombination with the PIB sequence of a group 3 strain. The PIB gene analysis of $1 \mathrm{~kb}$ of DNA represents only c. $0 \cdot 1 \%$ of the bacterial genome and differences outside this region cannot be detected. In contrast, PFGE examines the entire genome and can, therefore, potentially detect more variation.

Comparison of the amino-acid sequences of all IB-3 strains, which were recognised by a panel of monoclonal antibodies (MAbs) comprising MAbs 3C8 ('a'), 1F5 ('b') and 2H1 ('k'), and all IB-7 strains, which were recognised by a panel of MAbs comprising 2D6 ('c'), 2G2 (' $g$ ') and $2 \mathrm{H} 1$ (' $k$ '), indicated that the results from the present study concur with those from our previous study ${ }^{12}$ in which we proposed that codons $192,193,195,196$ and 198 were responsible for epitope recognition by MAb 3C8 (' $a$ '). Changes in $1-4$ of these amino acids did not prevent recognition by MAb $3 \mathrm{C} 8$ ('a'). In contrast to the other IB-3 strains, S21 had glutamine, asparagine, glutamine, isoleucine, valine and aspartic acid at positions 236-240. These positions have been proposed to be the epitope recognised by MAb 2D4 (' $h$ '), a MAb which does not recognise serovar IB-3 strains. ${ }^{12}$ A discrepancy thus emerged since strain S21 did not react with MAb 2D4 and hence was typed as serovar IB-3. Furthermore, strain S1 had amino-acid residues tyrosine, aspartic acid, glutamine, alanine and serine at positions 192, 193, 195, 196 and 198, respectively, corresponding to the epitope for MAb $3 \mathrm{C}^{12}$ but was typed as serovar IB-7 which should not react with $\mathrm{MAb} 3 \mathrm{C} 8$. These two discrepancies could be due to the subjective nature of interpretation of co-agglutination results from weak reactions or could have arisen from conformational hindrance caused by reactive epitopes, thereby preventing optimal recognition and binding of the MAbs.

The present study showed that despite the majority of strains being grouped within the same serovar, they could still be differentiated on the basis of PIB 
nucleotide and amino-acid sequence differences, especially at the epitope regions. The high correlation observed between PFGE, nucleotide and deduced amino-acid sequence analysis indicated that PFGE is a highly discriminatory tool, which may be more suitable for strain characterisation of $N$. gonorrhoeae for

\section{References}

1. Kihlström E, Danielsson D. Advances in biology, management and prevention of infections caused by Chlamydia trachomatis and Neisseria gonorrhoeae. Curr Opin Infect Dis 1994; 7: 25-33.

2. Kohl PK, Ison CA, Danielsson D, Knapp JS, Petzoldt D Current status of serotyping of Neisseria gonorrhoeae. Eur $J$ Epidemiol 1990; 6: 91-95.

3. Poh CL, Ocampo JC, Sng EH, Bygdeman SM. Characterisation of PPNG and non-PPNG Neisseria gonorrhoeae isolates from Singapore. Genitourin Med 1991; 67: 389-393.

4. Sarafian SK, Knapp JS. Molecular epidemiology of gonorrhea. Clin Microbiol Rev 1989; 2 Suppl: S49-S55.

5. Lau QC, Chow VTK, Poh CL. Polymerase chain reaction and direct sequencing of Neisseria gonorrhoeae protein IB gene: partial nucleotide and amino acid sequence analysis of strains S4, S11, S48 (serovar IB 4) and S34 (serovar IB 5). Med Microbiol Immunol 1993; 182: 137-145.

6. Poh CL, Lau QC. Subtyping of Neisseria gonorrhoeae auxotypeserovar groups by pulsed-field gel electrophoresis. $J \mathrm{Med}$ Microbiol 1993; 38: 366-370.

7. Butt NJ, Lambden PR, Heckels JE. The nucleotide sequence of the por gene from Neisseria gonorrhoeae strain $\mathrm{P} 9$ encoding epidemiological studies than direct PCR sequencing of the protein IB gene.

We thank S. Sarafian and J. Knapp for A/S typing of the gonococcal strains. This work was supported by National University of Singapore Research grants RP 910459 and RP 920320. Q.C. L. is an NUS Research Scholar.

outer membrane protein PIB. Nucleic Acids Res 1990; 14: 4258

8 Butt NJ, Virji M, Vayreda F, Lambden PR Heckels JE. Gonococcal outer-membrane protein PIB: comparative sequence analysis and localization of epitopes which are recognized by type-specific and cross-reacting monoclonal antibodies. J Gen Microbiol 1990; 136: 2165-2172

9. Maslow JN, Mulligan ME, Arbeit RD. Molecular epidemiology: application of contemporary techniques to the typing of microorganisms. Clin Infect Dis 1993; 17: 153-164.

10 Prevost G, Jaulhac B, Piemont Y. DNA fingerprinting by pulsed-field gel electrophoresis is more effective than ribotyping in distinguishing among methicillin-resistant Staphylococcus aureus isolates. J Clin Microbiol 1992; 30: 967-973.

11 Johnson WM, Bernard K, Marrie TJ, Tyler SD. Discriminatory genomic fingerprinting of Legionella pneumophila by pulsed-field electrophoresis. J Clin Microbiol 1994; 32: $2620-2621$

12. Chow VTK, Lau QC, Poh CL. Mapping of serovar-specific monoclonal antibody epitopes by DNA and amino acid sequence analysis of Neisseria gonorrhoeae outer membrane protein IB strains. Immunol Infect Dis 1994; 4: 202-206. 